\title{
Ensaio clínico placebo-controlado com isoflavonas da soja para sintomas depressivos em mulheres no climatério
}

Placebo-controlled clinical trial with soy isoflavones for depressive symptoms in climacteric women

Rilva Lopes de Sousa ${ }^{1}$, Rosália Gouveia Filizola ${ }^{2}$, Margareth de Fátima Formiga Melo Diniz ${ }^{3}$, Eduardo Sérgio Soares Sousa ${ }^{4}$, João Leonardo Ribeiro de Moraes ${ }^{5}$

\section{RESUMO}

Objetivos: avaliar a eficácia do uso de isoflavonas da soja no tratamento de sintomas depressivos em mulheres com síndrome climatérica. Métodos: estudo experimental placebo-controlado, randomizado e duplo-cego, com 84 pacientes climatéricas atendidas ambulatorialmente no Hospital Universitário Lauro Wanderley, em João Pessoa (PB). Na avaliação de sintomas depressivos empregou-se o Questionário de Auto-avaliação da Escala de Hamilton para Depressão (QAEH-D) nas visitas pré-tratamento (VT1) e na $8^{\mathrm{a}}(\mathrm{VT} 2)$ e na $16^{\mathrm{a}}$ (VT3) semana pós-tratamento. O grupo experimental (GExp) recebeu extrato de isoflavonas da soja, $120 \mathrm{mg}$ por dia, e o controle (GCont), placebo. A comparação dos escores do QAEH-D entre os grupos em VT1, VT2 e VT3 constituiu a medida primária de eficácia (teste $t, \mathrm{p} \leq 0,05$ ). Análise secundária incluiu estimativa do "efeito dominó" e avaliação clínica e laboratorial de eventos adversos. Resultados: houve redução significativa dos escores do QAEH-D no GExp (VT2<VT1; p=0,003), com redução adicional entre VT2 e VT3 (VT3<VT2; p=0,005). Similarmente, houve decréscimo dos escores entre VT1 e VT2 no GCont $(\mathrm{VT} 2<\mathrm{VT} 1 ; \mathrm{p}=0,04)$, que não persistiu posteriormente $(\mathrm{VT} 2=\mathrm{VT} 3$; p $>0,05)$. De VT1 para VT3, evidenciou-se diferença estatisticamente significativa de $8,9 \%$ na redução dos escores entre os grupos $(\mathrm{p}=0,03)$. Não houve correlação da redução dos sintomas depressivos com resposta dos sintomas vasomotores $(p>0,05)$. Houve redução das concentrações de FSH apenas no $\operatorname{GExp}(p=0,02)$, sem alterações do estradiol. Não ocorreram eventos adversos clinicamente relevantes. Conclusões: o efeito do extrato de isoflavonas foi superior ao do placebo, porém de pequena magnitude e apenas após 8 semanas de tratamento. Este pequeno efeito atribuído ao tratamento experimental, de boa tolerabilidade, poderá beneficiar pacientes que têm efeitos colaterais aos estrógenos ou que preferem não usar estes hormônios.

PALAVRAS-CHAVE: Climatério; Depressão; Isoflavonas; Estrogênios não esteróides; Saúde da mulher

\section{ABSTRACT}

Purpose: to evaluate the efficacy of the use of isoflavones in the treatment of depressive symptoms in climacteric women. Methods: placebo-controlled, randomized double-blind experimental study with 84 climacteric women who were assisted at the Lauro Wanderley University Hospital Ambulatory, in João Pessoa, Paraíba, Brazil. In the evaluation of the depressive symptoms the Self-evaluation questionnare of Hamilton's rating scale for depresion (QAEH-D) was used in the pretreatment visit (VT1), and in the 8th(VT2) and 16th(VT3) week after treatment. The experimental group (GExp) received soy extract with isoflavones, $120 \mathrm{mg}$ per day, and the control group (GCont), placebo. The comparison of the scores of the QAEH-D between the VT1, VT2 and VT3 groups constituted the primary measure of efficacy $(t$ test, $\mathrm{p} \leq 0.05)$. Secondary analysis included the estimate of the "domino hypothesis" and the clinical and laboratory evaluation of side effects. Results: there was a significant reduction of the QAEH-D scores in the GExp (VT2 $<\mathrm{VT} 1 ; \mathrm{p}=0,003)$, with additional reduction between VT2 and VT3 (VT3 $<$ VT2; $p=0.005)$. Similarly, there was a decrease of the scores between VT1 and VT2 in the GCont (VT2 $<$ VT1; $\mathrm{p}=0.04)$, which did not persist later $(\mathrm{VT} 2=\mathrm{VT} 3 ; \mathrm{p}>0.05)$. From VT1 to VT3, there was a significant difference $(8.9 \%)$ in the reduction of the scores between GExp and GCont $(\mathrm{p}=0.03)$. There was no correlation between the reduction of the depressive

Trabalho desenvolvido no Ambulatório de Climatério do Hospital Universitário Lauro Wanderley - Universidade Federal da Paraíba - UFPB - João Pessoa (PB), Brasil.

1 Doutoranda do Programa de Pós-Graduação em Produtos Naturais e Sintéticos Bioativos - Laboratório de Tecnologia Farmacêutica - Universidade Federal da Paraíba - UFPB - João Pessoa (PB), Brasil.

2 Professora de Endocrinologia do Departamento de Medicina Interna - Centro de Ciências da Saúde - Universidade Federal da Paraíba - UFPB - João Pessoa (PB), Brasil.

3 Professora do Programa de Pós-Graduação em Produtos Naturais e Sintéticos Bioativos - Laboratório de Tecnologia Farmacêutica - Centro de Ciências da Saúde - Universidade Federal da Paraíba - UFPB - João Pessoa (PB), Brasil.

4 Professor de Ginecologia do Departamento de Obstetrícia e Ginecologia - Centro de Ciências da Saúde - Universidade Federal da Paraíba - UFPB João Pessoa (PB), Brasil.

5 Professor de Psiquiatria do Departamento de Medicina Interna-Centro de Ciências da Saúde-Universidade Federal da Paraíba-UFPB - João Pessoa (PB), Brasil. Correspondência: Rilva Lopes de Sousa

Rua Isidro Gomes, 435, ap. 401 - Tambaú - 580160-39 - João Pessoa - PB - Telefone: (83) 3247-2842 - 9978-5437 - e-mail: rilvalopes@hotmail.com 
symptoms and alterations of the vasomotor symptoms ( $>0.05)$. There was a reduction of FSH concentrations only in GExp $(p=0.02)$, without estradiol modifications. Side effects were not clinically relevant. Conclusions: the effect of soy isoflavones was superior to the placebo, however, of small magnitude and only identified after 8 weeks of treatment. This small effect attributed to the experimental treatment, of good tolerability, may benefit patients who have side effects due to estradiol, or those who prefer not to use hormones.

KEYWORDS: Climacteric; Depression; Isoflavones; Estrogens, non-steroidal; Women's health

\section{Introdução}

$\mathrm{Na}$ última década tem crescido o interesse no papel dos estrógenos na melhora do humor e sensação de bem-estar psicológico em mulheres climatéricas não deprimidas, presumindo-se ser este efeito resultante da sua ação sobre a neurotransmissão cerebral ${ }^{1,2}$. A hipótese do efeito positivo dos estrógenos sobre sintomas depressivos foi corroborada por vários estudos ${ }^{3-5} \mathrm{e}$ em nosso ensaio clínico anterior ${ }^{6}$ no Brasil. Atualmente, em virtude das controvérsias relacionadas à estrogenioterapia, justifica-se o interesse em avaliar o efeito de compostos vegetais com bioatividade estrogênica, porém desprovidos de muitos dos efeitos adversos dos estrógenos.

Foram publicados vários estudos sobre os efeitos de extratos de isoflavonas da soja sobre a sindrome climatérica, focalizando como desfecho clínico os sintomas vasomotores, ou os sintomas climatéricos medidos por índices menopausais ${ }^{7-9}$. Entretanto, até o momento, nenhum estudo abordou o efeito de isoflavonas da soja sobre a sintomatologia depressiva como variável primária de pesquisa.

Assim, o objetivo deste trabalho foi avaliar eficácia e segurança de um extrato de isoflavonas da soja sobre a sintomatologia depressiva de mulheres com síndrome climatérica.

\section{Métodos}

\section{População, elegibilidade e local da pesquisa}

Este estudo experimental, com desenho de ensaio clínico placebo-controlado, duplo-cego e randomizado, incluiu 84 pacientes do sexo feminino com idade entre 45 e 60 anos atendidas no Ambulatório de Climatério do Hospital Universitário Lauro Wanderley (HULW), da Universidade Federal da Paraíba (UFPB), em João Pessoa, Paraíba (PB).

A seleção das pacientes ocorreu por meio de amostragem por conveniência. O tamanho da amostra foi calculado em 84 pacientes, com base na suposição de que o tratamento de sintomas depressivos seria considerado eficaz se resultasse na redução de $50 \%$ nos escores pré-tratamento de uma escala de auto-avaliação desses sintomas, detectando-se como relevante uma diferença de $20 \%$ entre o grupo experimental e o de controle, com significância estatística de $5 \%(p=0,05)$ em teste de hipótese bicaudal e $80 \%$ de poder estatístico.

Foram adotados os seguintes critérios de inclusão: idade entre 45 e 60 anos; um ano ou mais de amenorréia para mulheres não histerectomizadas; relato de ao menos três ondas de calor por dia, de intensidade moderada ou acentuada; niveis plasmáticos de hormônio folículo-estimulante (FSH) maiores ou iguais a $25 \mathrm{UI} / \mathrm{L}$; escolaridade mínima necessária para entendimento do questionário; e concordância por escrito em participar do estudo. Para caracterização do estado pós-menopausal em pacientes histerectomizadas, considerou-se o nivel de FSH plasmático ${ }^{9}$.

Foram excluídas as mulheres com uma ou mais das seguintes condições: ausência de pontuação em escala de avaliação de sintomas depressivos (Questionário de Auto-Avaliação da Escala de Hamilton para Depressão, QAEH-D) ${ }^{10}$; uso de medicamentos psicoativos durante o mês antes do início do estudo; tratamento com estrógenos, fitoestrógenos e moduladores seletivos sintéticos de receptores estrogênicos nos seis meses antes do início do estudo; diagnóstico de câncer ginecológico; doenças intestinais, hepáticas e/ou renais; transtornos do humor; psicoterapia em curso; niveis plasmáticos anormais do hormônio tiroestimulante; uso de antibióticos orais nos últimos dois meses; consumo regular de bebidas alcoólicas; e as vegetarianas.

\section{Procedimentos}

O projeto do estudo recebeu parecer favorável do Comitê de Ética em Pesquisa do HULW/UFPB (Processo número 331/04). As pacientes elegiveis foram informadas sobre o tratamento, sendo in- 
cluídas no estudo após assinar o termo de consentimento livre e esclarecido.

Realizaram-se quatro visitas durante o estudo: 1) visita de seleção (VS); 2) visita inicial de tratamento (VT1), na linha de base; 3) primeira visita de seguimento (VT2), oito semanas depois do início do tratamento; e 4) visita pós-tratamento final (VT3), 16 semanas após VT1. A técnica de coleta de dados foi a entrevista estruturada, constituída por perguntas fechadas da escala de sintomas depressivos (QAEH-D) e de um formulário de dados clínicos elaborado pelos autores.

$\mathrm{Na}$ VS, as mulheres que preencheram os critérios de idade, amenorréia e relato de ondas de calor foram entrevistadas com aplicação da escala QAEH-D. Obtendo escore diferente de zero e inferior ou igual ao ponto de corte de 16 , as voluntárias passaram à avaliação complementar. As pacientes que alcançaram o ponto de corte na aplicação do QAEH-D na VS foram submetidas a entrevista psiquiátrica por profissional de saúde mental, com o objetivo de excluir a possibilidade de transtornos do humor de acordo com a Classificação de Transtornos Mentais e de Comportamento da Organização Mundial de Saúde ${ }^{11}$, na CID-10.

$\mathrm{Na}$ avaliação complementar, realizaram-se os exames preconizados pela North American Menopause Society ${ }^{12}$. Como parte da avaliação de segurança do tratamento, foram também realizados hemograma, bioquímica sangüínea (glicemia, colesterol, triglicerídeos, transaminases, uréia e creatinina, bilirrubinas). Realizaram-se dosagens hormonais plasmáticas (estradiol e FSH). Os níveis de FSH foram dosados por método imunoradiométrico e os de estradiol por radioimunoensaio, usando-se o kit Coat-A-Count ${ }^{\mathrm{R}}$, da DPC (Diagnostic Products Corporation, Los Angeles, CA).

Na VT1, as pacientes elegiveis foram submetidas a nova entrevista com aplicação do QAEH$\mathrm{D}$, sendo registradas também a freqüência e a intensidade dos sintomas vasomotores. Em VT2 e VT3, repetiu-se a mesma avaliação feita em VT1, além da investigação de eventuais efeitos adversos. Em VT3, além dos procedimentos anteriores, também foram repetidos os exames hematológicos, bioquímicos e hormonais realizados antes do início do tratamento.

\section{Tratamento experimental}

As pacientes elegiveis foram designadas para um dos dois grupos (experimental ou controle) por alocação randômica simples. As pacientes do grupo experimental receberam $176 \mathrm{mg}$ de extrato de soja por dia (Isoflavin Beta ${ }^{\circledR}$, Galena, Campinas, SP), contendo $120 \mathrm{mg}$ de isoflavonas, divididos em duas doses de $60 \mathrm{mg}$, via oral, ao passo que as do grupo controle receberam duas doses diárias de placebo (amido).

Cada cápsula continha $88 \mathrm{mg}$ de extrato de soja, com $60 \mathrm{mg}$ de isoflavonas, na concentração de $68 \%$, sendo $20 \mathrm{mg}$ de daidzeína-daidzina, $17 \mathrm{mg}$ como daidzeína, $14 \mathrm{mg}$ como genisteína-genistina e $9 \mathrm{mg}$ como genisteína. O extrato seco de soja e o placebo foram encapsulados na Farmácia Magistral Dilecta, em João Pessoa (PB), sob encomenda da pesquisadora responsável. Os frascos numerados em código por uma farmacêutica não envolvida diretamente no estudo foram doados às pacientes nas visitas VT1 e VT2. A adesão ao tratamento foi monitorada pela contagem de cápsulas nas visitas VT2 e VT3.

\section{Desfecho clínico}

O instrumento de coleta dos dados da variável primária foi o QAEH-D, escala de auto-relato desenvolvida para medir sintomas depressivos na população geral, originada da Escala de Avaliação de Depressão de Hamilton ${ }^{10}$. O QAEH-D tem 18 itens, com uma pontuação global possivel entre 0 e 40. Demonstrou-se que o QAEH-D é medida válida e fidedigna de sintomas depressivos em nossa população ${ }^{6,13}$.

Análise secundária de eficácia incluiu o efeito sobre sintomas vasomotores e sua relação com a melhora de sintomas depressivos. O registro da freqüência dos sintomas vasomotores foi feito a partir de um diário preenchido pela própria paciente ao final do dia na última semana antes das visitas de avaliação de sintomas (VT1, VT2, VT3), sendo contabilizada a freqüência média dos sete dias de anotação.

A análise de segurança consistiu em avaliação clínica e laboratorial de eventos adversos. Os eventos adversos clínicos foram registrados de forma dicotômica ( $\mathrm{sim} /$ não), com perguntas referindo-se aos seguintes sintomas e sinais: diarréia, náuseas, vômitos, excesso de gases no abdome, ou outros (descrição solicitada). Foram considerados eventos adversos quaisquer ocorrências médicas, como resposta incoveniente e não intencional associada ao tratamento, ainda que não necessariamente apresentando relação causal com este, porém não atribuídos à evolução do climatério sintomático.

Entre as variáveis demográficas, a classificação econômica foi determinada pelo questionário CCEB (Critério de Classificação Econômica Brasil) da Associação Brasileira das Empresas de Pesquisa ${ }^{14}$.

\section{Análise estatistica}

A medida primária de eficácia consistiu na comparação entre os grupos experimental (GExp) e controle (GCont) da redução percentual dos es- 
cores entre as avaliações de eficácia (VT2 e VT3) em relação aos escores iniciais do QAEH-D (VT1), pelo teste $t$ de Student para amostras independentes. O teste $t$ para amostras emparelhadas complementou a análise, com a comparação dos escores médios entre as avaliações (VT1-VT2, VT2-VT3, VT1-VT3).

A avaliação do desfecho foi feita mediante cálculo da variação percentual das pontuações ${ }^{15}$, através da fórmula $\Delta \%=($ escore VT 1 - escore VT3) / (escore VT1) x 100. O tamanho do efeito foi calculado pela diferença média padronizada entre dois grupos (d de Cohen) usando a fórmula dos desviospadrão combinados ${ }^{16}$.

O teste $\chi^{2}$ (ou o teste exato de Fisher) foi usado para comparação da distribuição de variáveis categóricas, e a análise de correlação de Pearson ( $r)$ para avaliar a fidedignidade teste-reteste do QAEH-D (mensurações de VS e VT1), bem como a verificação de correlações entre escores do QAEH$\mathrm{D}$ e a freqüência dos sintomas vasomotores. As análises estatísticas foram feitas com uso do Statistical Package for Social Sciences (SPSS, Chicago), versão 11.5 para Windows. Na análise de tolerância, considerou-se o número total de pacientes randomizadas $(n=84)$ e que iniciaram o tratamento.

\section{Resultados}

\section{Recrutamento e seleção da amostra}

Entre as 191 pacientes atendidas na VS, 96 foram excluídas na VS (58 não preencheram todos os critérios de exclusão e 38 preencheram um ou mais critérios de exclusão). Das 22 pacientes que alcançaram o ponto de corte de 10 na escala QAEHD, 14 (63,6\%; 14/22) apresentavam transtornos do humor, ao passo que uma (1) recebeu outro diagnóstico da CID-10. As outras sete pacientes que alcançaram o ponto de corte, mas não preencheram critérios diagnósticos da CID-10, foram incluídas no estudo.

Das 95 pacientes elegiveis na VT1, 84 foram convidadas a participar da fase experimental, encerrando-se a inclusão de novas pacientes quando se alcançou esse número. Houve perda de cinco pacientes durante a fase experimental, completando o tratamento de 16 semanas 79 pacientes, o que representa 94\% da amostra que sofreu randomização.

\section{Caracteristicas das pacientes na linha de base}

A idade das 84 pacientes incluídas no estudo experimental variou entre 45 e 60 anos $(85,7 \%$ entre 50 e 60 anos), com média de $53,3( \pm 3,6)$ anos. Cinqüenta e quatro mulheres $(64,3 \%)$ eram casadas; $44(52,3 \%)$ pardas ou negras; $61(72,6 \%)$ tinham instrução formal entre básica e média completa; $73(86,9 \%)$ pertenciam às classes econômicas média-média e média-baixa e $43(51,2 \%)$ não exerciam atividade remunerada. GExp e GCont foram homogêneos quanto à distribuição destas variáveis sociodemográficas. Não houve diferenças estatísticas na comparação dos níveis plasmáticos de estradiol e FSH entre os grupos.

\section{Efeito do tratamento sobre os escores do QAEH-D}

Os escores obtidos por meio do QAEH-D pela amostra $(n=84)$ variaram entre 3 e 19 pontos, com média de $8,3( \pm 3,1)$. Não houve diferença entre os escores médios de QAEH-D entre os dois grupos na linha de base $(p>0,05)$.

$\mathrm{Na}$ avaliação de eficácia, a comparação das pontuações obtidas com o QAEH-D também não revela diferença estatisticamente significativa en-

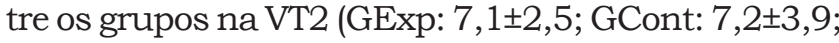
p $>0,05$ ) e na VT3 (GExp: 5,8 $\pm 2,2$; GCont: 6,3 $\pm 3,0$; $p>0,05)$. As diferenças dos escores médios entre as avaliações de eficácia VT1-VT2, VT2-VT3 e VT1VT3 estão na Tabela 1. Comparando GExp entre VT1 e VT2, observa-se que houve redução significativa dos escores do QAEH-D (VT2<VT1). Tal redução teve um incremento adicional também significativo entre as avaliações VT2 e VT3 (VT3<VT2). Também no GCont, observou-se redução significativa nos escores dos sintomas depressivos entre VT1 e VT2 (VT2<VT1), porém neste grupo não houve redução adicional entre VT2 e VT3 (VT2=VT3).

Tabela 1 - Diferenças das médias dos escores do QAEH-D entre as avaliações de eficácia em relação à linha de base.

\begin{tabular}{|c|c|c|c|c|}
\hline \multirow[t]{2}{*}{ Grupos } & \multirow[t]{2}{*}{ Parâmetros } & \multicolumn{3}{|c|}{$\begin{array}{l}\text { Variação dos escores do QAEH-D } \\
\text { entre as avaliações }\end{array}$} \\
\hline & & VT1-VT2 & VT2-VT3 & VT1-VT3 \\
\hline \multirow[t]{5}{*}{$\overline{G E x p}$} & $\mathrm{~m}$ & 1,4 & 1,4 & 2,8 \\
\hline & $\mathrm{s}$ & 2,1 & 2,0 & 2,0 \\
\hline & IC $95 \%$ & $0,7-2,0$ & $0,7-2,0$ & $2,2-3,4$ \\
\hline & $p$ & $0,003^{*}$ & $0,005^{*}$ & $0,001^{*}$ \\
\hline & gl & 40 & 39 & 39 \\
\hline \multirow[t]{5}{*}{ GCont } & $\mathrm{m}$ & 0,9 & 0,6 & 1,7 \\
\hline & $s$ & 2,61 & 2,56 & 2,69 \\
\hline & IC 95\% & $0,1-1,7$ & $-0,2-1,4$ & $0,9-2,6$ \\
\hline & $p$ & $0,001^{*}$ & 0,30 & $0,002^{*}$ \\
\hline & gl & 41 & 38 & 38 \\
\hline
\end{tabular}

QAEH-D: Questionário de Auto-Avaliação da Escala de Hamilton para Depressão. m médias; s: desvios-padrão; gl: graus de liberdade; IC 95\%: intervalos de confiança de 95\%; p: nível de significância.

VT1 = $1^{\text {a }}$ visita de tratamento ou de linha de base; VT2 $=2^{a}$ visita de tratamento ou avaliação intermediária na $8^{\mathrm{a}}$ semana da fase experimental; VT3 $=3^{\mathrm{a}}$ visita de tratamento ou avaliação final na $16^{a}$ semana da fase experimental.

${ }^{*}$ Estatisticamente significativo: $p<0,05$, teste $t$ de Student para amostras dependentes. 
Para analisar tais diferenças, as mudanças nos escores do QAEH-D foram convertidos em variação percentual $(\Delta \%)$ entre VT1-VT2, VT2-VT3 e VT1-VT3 nos dois grupos para determinação do tamanho do efeito, como mostra a Figura 1. As variações percentuais quanto às mudanças das pontuações de QAEH-D dos grupos GExp e GCont não alcançaram diferença estatística entre si nas medições de VT1 para VT2 e de VT2 para VT3. Porém, diferença estatisticamente significativa foi observada entre os dois grupos quando se compararam as reduções percentuais entre VT1 e VT3, ou seja, entre a linha de base e a avaliação final após 16 semanas de tratamento, com uma diferença percentual entre os grupos de $8,9 \%$. O tamanho do efeito calculado (d de Cohen) foi de 0,4.

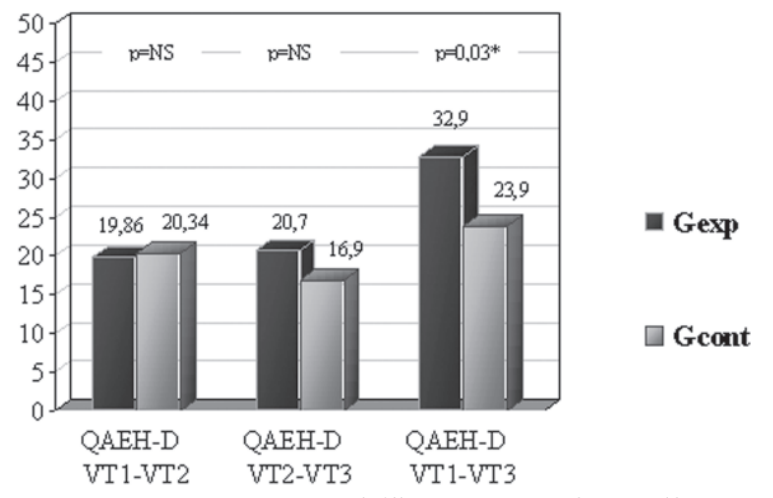

Figura 1 - Reduções percentuais médias $(\Delta \%)$ dos escores do QAEH-D (Questionário de Auto-Avaliação da Escala de Hamilton para Depressão) nos intervalos entre as avaliações ou visitas de tratamento (VT1-VT2, VT2-VT3, VT1-VT3) nos grupos experimental (GExp) e controle (GCont). "Estatisticamente significativo. NS: não significativo estatisticamente $(p>0,05)$.

Melhora considerada significativa do ponto de vista clínico ( $\Delta \geq 50 \%$ de redução dos escores) nos escores na VT3 em comparação com a VT1 ocorreu em 11 (11/40; 27,5\%) no GExp, enquanto no GCont foi observada em apenas 5,1\% (2/39) das pacientes. Esta diferença foi estatisticamente significativa de acordo com o teste exato de Fisher $(\mathrm{p}=0,01)$.

Convertendo as mudanças percentuais $(\Delta \%)$ dos escores do QAEH-D entre VT1 e VT3 em variáveis categóricas, definiram-se três niveis ordinais de variável dependente: "resposta sintomatológica completa" $(\Delta \% \geq 50 \%)$, "resposta parcial" (redução< $50 \%)$ e ausência de resposta $(\Delta \%=0)$. Resposta clínica completa foi mais freqüente no GExp, ocorrendo em $11(27,5 \%)$ pacientes, ao passo que ocorreu em apenas $2(5,12 \%)$ no GCont. A ausência de resposta à intervenção foi observada em duas (2/ $40 ; 5 \%)$ pacientes do GExp e em sete $(7 / 39 ; 17,2 \%)$ do GCont $(p>0,05)$. Pelo teste $\chi^{2}$, verificou-se que as distribuições das freqüências de pacientes em cada uma dessas categorias diferiram significativamente entre os grupos $(p=0,01)$.

\section{Efeito do tratamento sobre os sintomas va- somotores e relação com a evolução dos sinto- mas depressivos}

De forma semelhante ao que ocorreu com os escores do QAEH-D, o número médio diário de ondas de calor apresentou redução estatisticamente significativa entre VT1 e VT2 no GExp e no GCont, sem diferença entre os grupos, como evidencia a Tabela 2. Durante as oito semanas subseqüentes, entretanto, posterior redução estatisticamente significativa ocorreu no $\operatorname{GExp}(p=0,001)$, mas não no GCont ( $p>0,05)$. Comparando-se as reduções percentuais médias de VT3 em relação a VT1 entre GExp e GCont, evidencia-se uma di-

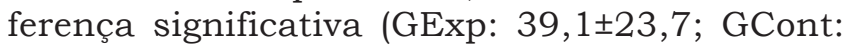
$27,1 \pm 19,5 ; p=0,01)$.

Tabela 2 - Comparação da frequêencia e intensidade de ondas de calor nos grupos experimental (GExp) e controle (GCont).

\begin{tabular}{llcccc}
\hline Avaliações & Ondas de calor & \multicolumn{2}{c}{$\begin{array}{c}\text { Estatística } \\
\text { descritiva }\end{array}$} & \multicolumn{2}{c}{$\begin{array}{c}\text { Estatística } \\
\text { inferencial* }\end{array}$} \\
& & GExp & GCont & & \\
& & $\mathbf{m} \pm \mathbf{s}$ & $\mathbf{m} \pm \mathbf{s}$ & p & gl \\
\hline VT1 & freqüência & $7,0 \pm 3,6$ & $6,5 \pm 2,8$ & NS & 82 \\
& escore compósito & $19,3 \pm 12,1$ & $18,7 \pm 9,3$ & NS & 82 \\
VT2 & freqüência & $5,5 \pm 2,5$ & $5,0 \pm 2,8$ & NS & 81 \\
& escore compósito & $9,3 \pm 7,8$ & $9,9 \pm 7,8$ & NS & 81 \\
VT3 & freqüência & $4,1 \pm 2,3$ & $4,7 \pm 2,2$ & NS & 77 \\
& escore compósito & $6,2 \pm 5,3$ & $7,3 \pm 5,2$ & NS & 77 \\
\hline
\end{tabular}

m: médias; s: desvios-padrão; gl: graus de liberdade. VT1 = ${ }^{\text {a }}$ visita de tratamento ou de linha de base; VT2 $=2^{\mathrm{a}}$ visita de tratamento ou avaliação intermediária na $8^{\mathrm{a}}$ semana da fase experimental; VT3 $=3^{\mathrm{a}}$ visita de tratamento ou avaliação final na $16^{\mathrm{a}}$ semana da fase experimental.

*Teste $t$ para amostras independentes; NS: não significativo estatisticamente $(p>0,05)$

Vinte cinco pacientes da amostra apresentaram resposta clinicamente significativa quanto à redução da freqüência das ondas de calor $(\Delta \geq 50 \%)$ após 16 semanas de tratamento. Destas, 18 eram do GExp (45\%; 18/40), ao passo que 7 eram do GCont $(17,9 \% ; 7 / 39)$, havendo diferença estatisticamente significativa $(\mathrm{p}=0,01)$, com uma proporção superior dessa resposta $(\Delta \geq 50 \%)$ no grupo experimental, em relação ao controle.

Não houve correlação entre redução percentual média dos escores do QAEH-D e redução percentual da freqüência de sintomas vasomotores entre VT1 e VT3 no GExp ( $p>0,05)$, nem no GCont $(p>0,05)$. A redução da freqüência das ondas de calor também não mostrou associação com as reduções dos escores do QAEH-D entre VT1 e VT2 ou entre VT2 e VT3 $(p>0,05)$. 


\section{Efeito sobre concentrações hormonais de estradiol e FSH}

As concentrações de FSH apresentaram redução significativa entre as dosagens pré-tratamento e pós-tratamento $(\mathrm{p}=0,02)$ no GExp (Tabela 3). Quanto aos níveis plasmáticos de estradiol, embora se tenha observado pequena elevação da avaliação pré-tratamento $(13,0 \pm 7,1 \mathrm{pg} / \mathrm{mL})$ para a pós-tratamento $(16,7 \pm 15,2 \mathrm{pg} / \mathrm{mL})$ no GExp, esta alteração não chegou a alcançar significação estatística $(\mathrm{p}>0,05)$.

Tabela 3 - Comparação das concentrações de hormônio folículo-estimulante (FSH) e estradiol nas dosagens pré-tratamento e pós-tratamento nos grupos experimental (GExp) e controle (GCont)

\begin{tabular}{llcccc}
\hline Variáveis & Grupos & $\begin{array}{c}\mathbf{n} \\
\end{array}$ & $\begin{array}{c}\text { Pré-tratamento } \\
\text { VT1 }(\mathbf{m} \pm \mathbf{s})\end{array}$ & $\begin{array}{c}\text { Pós-tratamento } \\
\text { VT3 }(\mathbf{m} \pm \mathbf{s})\end{array}$ & $\begin{array}{c}\text { Valor } \\
\text { de } \mathbf{p}\end{array}$ \\
\hline FSH & GExp & $39^{*}$ & $80,76 \pm 29,19$ & $73,66 \pm 28,93$ & $0,02^{* *}$ \\
(mUl/mL) & GCont & $37^{*}$ & $73,62 \pm 30,3$ & $74,22 \pm 25,93$ & $\mathrm{NS}$ \\
Estradiol & GExp & 39 & $13,01 \pm 7,17$ & $16,74 \pm 15,27$ & $\mathrm{NS}$ \\
(pg/mL) & GCont & 37 & $14,13 \pm 7,48$ & $14,32 \pm 8,37$ & $\mathrm{NS}$
\end{tabular}

$\mathrm{VT} 1=1^{\text {a }}$ visita de tratamento ou de linha de base; VT3 $=3^{\text {a }}$ visita de tratamento ou avaliação final na $16^{\mathrm{a}}$ semana da fase experimental. GExp: grupo experimental; GCont: grupo controle; FSH = hormônio folículo-estimulante; m: médias; s: desvios-padrão; n: número de pacientes.

*A comparação dos pares de valores foi feita com o número de pacientes que tinham as dosagens pré- e pós-tratamento. ${ }^{* *}$ Estatisticamente significativo a 0,05 ; teste $t$ para amostras dependentes. NS: estatisticamente não significativo $(p>0,05)$

\section{Tolerância ao tratamento e eventos adversos}

O número de perdas ocorridas durante a fase experimental (pacientes que não completaram o tratamento) foi de cinco (5,9\%) nos dois grupos: duas no GExp $(4,7 \% ; 2 / 42)$ e três no GCont $(7,1 \%$; $3 / 42)$. As duas perdas do GExp ocorreram por ineficácia terapêutica $(n=1)$ e por efeito secundário $(n=1)$. Este último foi referido como cefaléia intensa após duas semanas de início do tratamento. No GCont, as perdas ocorreram por ineficácia terapêutica $(n=2)$. Perdeu-se o seguimento clínico da terceira paciente que saiu do estudo no GCont antes de seu término. As características das pacientes que se retiraram foram similares às daquelas que completaram o estudo.

Entre os eventos adversos relatados pelas pacientes que completaram o tratamento $(n=79)$, predominaram os sintomas de natureza gastrointestinal, os quais foram de leve intensidade e de curso transitório, não motivando nenhuma outra intervenção, sendo reportados por 12 pacientes do GExp (30\%; 12/40) e por sete pacientes do GCont $(17,9 \%)$. O teste exato de Fisher evidenciou que esta diferença na distribuição das freqüências de eventos gastrointestinais entre os grupos não foi estatisticamente significativa $(p>0,05)$. A Tabela 4 exibe a descrição destes e de outros eventos ci- tados pelas pacientes durante o tratamento. Não foram observadas alterações de relevância nos parâmetros hematológicos e dosagens bioquímicas pelo tratamento.

Tabela 4 - Descrição e freqüências dos eventos adversos relatados durante o ensaio ( $n=84)$.

\begin{tabular}{lcccc}
\hline Eventos & \multicolumn{2}{c}{ GExp } & \multicolumn{2}{c}{ GCont } \\
& $\begin{array}{c}(\mathbf{n}=\mathbf{4 2}) \\
\text { (n= }\end{array}$ & $\mathbf{f}$ ( $)$ & f & $\%$ \\
\hline Gastrointestinais & & & & \\
Cólica abdominal & 3 & 7,1 & - & - \\
Náuseas & 2 & 4,7 & 1 & 2,3 \\
Constipação intestinal & 2 & 4,7 & 3 & 7,1 \\
Diarréia & 2 & 4,7 & - & - \\
Epigastralgia & 2 & 4,7 & - & - \\
Flatulência & 1 & 2,3 & 2 & 7,1 \\
Boca seca & - & - & 1 & 2,3 \\
Outros eventos & & & & \\
Artralgias & 2 & 4,7 & 3 & 7,1 \\
Cefaléia & 2 & 4,7 & 2 & 4,7 \\
Aumento de apetite & 1 & 2,3 & 2 & 4,7 \\
Dor mamária & 1 & 2,3 & - & - \\
Tremores de extremidades & 1 & 2,3 & - & - \\
Tonturas & 1 & 2,3 & 1 & 2,3 \\
Visão turva & 1 & 2,3 & - & - \\
Insônia & 1 & 2,3 & - & - \\
Edema de membros inferiores & - & - & 1 & 2,3 \\
\hline
\end{tabular}

GExp: grupo experimental; GCont: grupo controle.

\section{Discussão}

A principal hipótese deste estudo foi a de que o tratamento com isoflavonas da soja produziria efeito superior ao do placebo sobre sintomas depressivos em mulheres climatéricas não deprimidas. Esta hipótese foi elaborada por analogia em relação a nosso estudo anterior, no qual se testou o efeito de estrógenos conjugados sobre sintomas depressivos em pacientes com sindrome climatérica atendidas no mesmo serviço de saúde ${ }^{6}$.

Naquele estudo, o tratamento levou a melhora sintomatológica significativa não relacionada ao alivio dos sintomas vasomotores, presumindo-se, então, a existência de possivel ação estrogênica positiva sobre a neurotransmissão cerebral. Supôsse que as isoflavonas produziriam resultado semelhante, por sua ação como agonistas estrogênicos parciais na presença de carência estrogênica pósmenopausal; além disso, tal resposta não constituiria apenas um "efeito placebo", mas seria relacionada à sua ação estrógeno-mimética. 
Trata-se de problema de pesquisa plausivel, com fundamento teórico, mas ainda não testado empiricamente. As isoflavonas exerceriam efeitos similares aos dos estrógenos por sua ação sobre receptores estrogênicos (RE) presentes no sistema nervoso central, pois têm maior afinidade de ligação com o RE $\beta$, o qual tem maior expressão no cérebro que o RE $\alpha$, sobretudo no hipocampo e córtex cerebral $^{17}$. Além disso, a afinidade diferencial das isoflavonas pelo RE $\beta$ é somente três vezes menor que a do $17-\beta$-estradiol, ao passo que a afinidade desses fitoquímicos pelo RE $\alpha$ é 1.000 a 10.000 vezes menor que a do estrógeno ${ }^{8}$. A premissa da possibilidade de efeitos benéficos desses compostos sobre sintomas psicológicos parte também de estudos com modelos animais, nos quais se demonstrou ação antidepressiva pelo uso de isoflavonas em fêmeas ooforectomizadas ${ }^{18,19}$.

Os resultados observados no nosso estudo indicam que houve redução significativa dos sintomas depressivos mensurados pelo QAEH-D no grupo experimental, superior à resposta do grupo controle, sugerindo que as isoflavonas podem ter um efeito sobre esses sintomas da síndrome climatérica. Tal redução superior à do placebo foi verificada somente na $16^{\mathrm{a}}$ semana de tratamento, indicando que há necessidade de tempo mais prolongado que oito semanas (avaliação de eficácia anterior) para se alcançar efeito superior àquele atribuído a fatores inespecíficos, sendo então, passivel de ser detectado clinicamente. A demora para obtenção de um efeito aparente das isoflavonas já foi relatada em relação aos sintomas vasomotores com o uso de estrógenos ${ }^{7,20}$.

Entretanto, ainda que estatisticamente significativa, a diferença entre a eficácia do grupo que recebeu isoflavonas em relação à do grupo controle foi pequena $(8,9 \%)$, sendo necessário considerar se esta diferença é clinicamente relevante. O tamanho do efeito (effect size) calculado ( $\mathrm{d}$ de Cohen $=0,4$ ) foi pequeno ${ }^{16}$, indicando que $66 \%$ das pacientes do GCont estão abaixo da média observada no GExp.

Um tamanho do efeito pequeno, como o que foi evidenciado, poderia ser clinicamente relevante se a condição para a qual o tratamento está sendo instituído é transtorno com repercussão clínica importante e sem outras possibilidades terapêuticas existentes. No entanto, comparar a relação custo-efetividade de tratamentos convencional e à base de fitomedicamentos para a sindrome climatérica é complexo, uma vez que, embora a terapia com estrógenos seja o tratamento mais eficaz para esse quadro, devem ser considerados também os eventos adversos da terapêutica hormonal.

Nesse sentido, levando em conta os custos indiretos na relação custo-benefício do tratamento com isoflavonas, é importante considerar tam- bém que mesmo o alivio parcial pode ser suficiente para algumas pacientes sintomáticas que preferem não receber estrógenos. Este efeito pequeno poderá beneficiar pacientes que decidem não usar a terapêutica hormonal ou que apresentam efeitos colaterais com o uso de estrógenos, tais como sangramento vaginal, náuseas, cefaléia, mastalgia, sinais de retenção hídrica e, no caso do esquema combinado com progesterona, alterações do humor, e para as quais outras alternativas medicamentosas não sejam apropriadas.

Por outro lado, há evidências de que as mulheres climatéricas estão seguindo uma tendência ao uso de medicamentos à base de plantas para seus sintomas menopausais ${ }^{21}$. Mesmo o alivio parcial pode ser suficiente, ou clinicamente relevante, considerando que as principais indicações do uso de isoflavonas no climatério atualmente são intolerância ou não-aceitação da terapia com estrógenos por parte da paciente. Esse subgrupo de mulheres poderia ser beneficiado por uma melhora parcial ${ }^{22}$, que foi a resposta mais freqüentemente encontrada no presente estudo.

$\mathrm{O}$ efeito placebo evidenciado tanto em relação aos sintomas depressivos quanto aos vasomotores mostrou magnitude compativel com a taxa de 20 a $30 \%$ para o tratamento de sintomas climatéricos ${ }^{23,24}$. A literatura indica que a administração de extrato de isoflavona de soja reduz os sintomas climatéricos em aproximadamente $45 \%$, deduzindo-se, então, que apenas 15 a $25 \%$ da redução desses sintomas são atribuídos ao efeito direto das isoflavonas ${ }^{24}$. Mesmo esta faixa de redução está acima da proporção observada na avaliação de eficácia do tratamento experimental sobre a variável primária do presente estudo, que focalizou primariamente sintomas depressivos.

No que concerne especificamente à avaliação do efeito de isoflavonas sobre os sintomas depressivos, não há outros estudos focalizando a eficácia destes compostos sobre a mesma variável para a comparação dos resultados do presente trabalho. De nosso conhecimento, este é o primeiro estudo em que os efeitos das isoflavonas da soja foram avaliados quanto ao seu impacto sobre os sintomas depressivos em pacientes no climatério. Os efeitos psicológicos das isoflavonas têm sido pouco investigados na literatura, tendo sido focalizados apenas como variável secundária em estudos observacionais com mulheres idosas ${ }^{25,26}$.

É importante salientar que o efeito placebo na resposta de sintomas climatéricos é descrita tanto em ensaios clínicos com estrógenos ${ }^{27,28}$, quanto em estudos com isoflavonas ${ }^{29,30}$. Este efeito placebo constitui uma resposta à intervenção médica, e apesar de ser um resultado da mesma, não tem relação com o mecanismo específico de ação farmacológica ${ }^{31}$. Tal efeito inespecífico 
marcante parece ser uma das razões que fazem com que muitos dos ensaios clínicos tenham sido incapazes de detectar efeitos pequenos pela ação estrogênica fraca das isoflavonas em relação aos sintomas climatéricos.

O efeito observado no grupo que recebeu placebo parece ter sido dissipado com o tempo, corroborando o que se afirma sobre respostas-placebo ${ }^{23}$. $\mathrm{Na}$ avaliação da $16^{\mathrm{a}}$ semana de tratamento o efeito observado no GCont foi superado pelo efeito do tratamento com isoflavonas. Não é possivel deduzir se as respostas às isoflavonas e ao placebo seriam superpostas, havendo efeito farmacológico pela ação agonista parcial das isoflavonas associado ao efeito inespecífico, não farmacológico, ou se estes efeitos ocorreriam de forma consecutiva.

Se são consecutivos, supõe-se que ambos poderiam ocorrer em uma mesma paciente, primeiro ocorrendo uma melhora atribuivel ao placebo e, depois, um efeito neurofisiológico das isoflavonas, que sustentaria a melhora clínica alcançada. Mesmo sendo independentes os efeitos atribuiveis à droga ativa e ao placebo, ficou evidente que proporção considerável da melhora observada com a droga ativa é decorrente de efeito placebo.

Por esta razão, o estudo deve ter uma duração suficientemente longa para que o efeito placebo inicial (que pode ocorrer tanto para sintomas fisicos quanto para sintomas psíquicos) possa desaparecer e a diferença entre grupos com droga ativa e placebo possa ser detectada clinicamente ${ }^{31}$. Admite-se que ensaios com desfechos clínicos de natureza subjetiva no climatério exigem duração de no mínimo seis semanas, embora seja recomedável que se estendam até 12 semanas para enfatizar o contraste entre efeitos do tratamento e o placebo, minimizando a possibilidade de erros tipo II, ou seja, aceitação da hipótese nula quando esta é falsa.

$\mathrm{O}$ pequeno efeito atribuído às isoflavonas no presente estudo deve ser discutido com relação a aspectos farmacológicos desses fitoquímicos. Diferenças individuais na absorção dos fitoestrógenos, niveis de estradiol no plasma, afinidade pelos receptores estrogênicos e distribuição tecidual destes receptores são fatores importantes na obtenção de uma resposta terapêutica satisfatória ${ }^{32}$.

Nesse aspecto, a mensuração dos niveis circulantes de isoflavonas nas participantes poderia prover informações sobre a absorção e biodisponibilidade destes compostos, mas esses dados não puderam ser determinados no presente estudo, não havendo, portanto, como demonstrar a magnitude em que houve efetiva absorção intestinal na amostra estudada. Os três estudos clínicos realizados no Brasil para avaliação dos efeitos de isoflavonas, tanto sob a forma de extrato de soja contendo isoflavonas $^{7}$, gérmen de soja contendo isoflavonas ${ }^{33}$ e pro- teína de soja ${ }^{34}$, também não realizaram dosagem de isoflavonas no plasma.

$\mathrm{Na}$ avaliação do efeito do tratamento com isoflavonas sobre sintomas depressivos, é necessário considerar também a influência da redução dos sintomas vasomotores sobre a resposta observada sobre aquela variável. Alguns autores consideram que a melhora de alterações do sono, ânimo depressivo e fadiga em pacientes climatéricas poderia resultar da diminuição dos sintomas vasomotores, o que repercutiria em melhor ânimo e sensação de bem estar, menos fadiga e nervosismo, conforme postula a hipótese do "efeito dominó" ${ }^{35,36}$. No entanto, a avaliação de coeficientes de correlação entre sintomas depressivos e vasomotores no presente estudo indicou que tais manifestações não se relacionaram linearmente nesta amostra.

Em estudo anterior também não encontramos evidências que corroborem esta hipótese ${ }^{37}$. $\mathrm{O}$ que parece ocorrer é que, sendo os sintomas vasomotores e depressivos ambos medidos por meio do auto-relato, tais manifestações, que devem estar subjacentes ao mesmo processo biológico, tendem a apresentar resposta semelhante.

No que se refere aos sintomas vasomotores, a literatura sugere que a administração de extrato de isoflavonas da soja reduz tais manifestações em cerca de $45 \%$ dos $\operatorname{casos}^{24}$, o que foi compativel com a proporção que encontramos neste estudo. Efeito de extrato de isoflavonas superior ao placebo sobre sintomas vasomotores foi demonstrado por quatro estudos anteriores ${ }^{23,38}$, nos quais foi usada a metade da dose empregada no nosso ensaio, com reduções sintomatológicas percentuais variando entre 10 e $40 \%$.

Por outro lado, dois outros estudos controlados com extrato de isoflavonas encontraram efeitos semelhantes ao do grupo placebo com doses de 75 a $80 \mathrm{mg}$ por dia ${ }^{10,39}$. Um efeito menor que o do estrógeno convencional, porém maior que o do placebo, foi observado com $54 \mathrm{mg} /$ dia de genisteína (24\%), menor que o efeito dos estrógenos (54\%) durante 12 meses $^{30}$. Efeito comparável ao do estrógeno convencional $(70 \%$ de melhora nos dois grupos) foi observado com $120 \mathrm{mg}$ de extrato de isoflavonas da soja comparado com estrógenos eqüinos conjugados em mulheres pós-menopáusicas brasileiras durante seis meses ${ }^{7}$.

Os demais estudos avaliaram o efeito de extrato com isoflavonas sobre sintomas vasomotores, mas não foram controlados, e outros ensaios publicados não usaram extratos, e sim proteína de soja contendo isoflavonas, gérmen de soja ou cereal de soja, com resultados dissonantes entre si.

O encontro de uma redução significativa nos niveis plasmáticos de FSH indica que houve efeito estrogênico no grupo que recebeu isoflavonas 
no presente estudo. Supõe-se que se um composto fitoestrogênico atua em vias de sinalização estrogênica como agonista, deveria haver diminuição dos níveis plasmáticos de FSH por causa do mecanismo de feedback negativo na hipófise, embora se suponha que haja subpopulações de mulheres que são mais sensiveis às isoflavonas que outras $^{9,37}$. Em concentrações de 100-1000 vezes as do estradiol, que são os niveis provavelmente encontrados no plasma humano após consumo regular de isoflavonas, estes compostos devem ser capazes de competir com estrógenos endógenos, ligando-se aos receptores estrogênicos, e interferir com a liberação de gonadotropinas ${ }^{40}$.

Quanto aos niveis plasmáticos de estradiol, embora se tenha observado pequena elevação na dosagem pós-tratamento no grupo que recebeu isoflavonas, esta alteração não chegou a alcançar significação estatística. Supõe-se que o consumo de isoflavonas afete o metabolismo dos estrógenos em algumas pacientes, alterando as concentrações plasmáticas desses hormônios ${ }^{31}$. Porém, os efeitos das isoflavonas sobre os niveis circulantes de estradiol, por si só, não são mecanismos explicativos dos seus efeitos clínicos em mulheres no climatério ${ }^{17,40}$.

No que concerne aos eventos adversos pesquisados neste ensaio, em vista da natureza esporádica das queixas relatadas nos dois grupos, sem diferença significativa entre si, não é possível determinar sua associação com o uso de isoflavonas. Outros estudos relataram tolerância excelente pelo uso de extrato de isoflavonas na dose de 40 a $50 \mathrm{mg}$ diários ${ }^{7,9,33,38}$. A falta de mudanças nos parâmetros bioquímicos indicam boa tolerabilidade da dose de $120 \mathrm{mg}$ usada pela nossa amostra, corroborando os dados de estudos anteriores com doses semelhantes ${ }^{7,34,38}$.

Concluímos que o tratamento com isoflavonas da soja levou a pequena redução dos sintomas depressivos, superando levemente a resposta do grupo controle apenas depois de 8 semanas de tratamento, sendo a redução sintomática inicial relacionada a uma resposta-placebo marcante, compativel com o que é descrito na literatura para o tratamento de sintomas climatéricos em geral.

Esse pequeno efeito sobre os sintomas depressivos atribuído às isoflavonas poderá, no entanto, beneficiar pacientes que decidem não usar estrógenos ou que apresentam efeitos colaterais pelo uso destes hormônios, para as quais mesmo o alivio parcial pode ser suficiente. A melhora dos sintomas depressivos não dependeu da redução dos vasomotores, contrariamente ao que postula a hipótese do efeito dominó. Houve boa tolerabilidade da dose de $120 \mathrm{mg}$ diários de isoflavonas, não havendo associação aparente entre os eventos adversos esporádicos observados e o tratamento.

\section{Agradecimentos}

Agradecemos às nossas alunas do curso de Medicina da UFPB, Débora Dantas de Albuquerque e Clarissa de Moura Souza, e aos médicos residentes de Ginecologia do HULW, Eguimar Fernandes Fïho e Jordana Pascoal Machado, pela ajuda na realização da propedêutica ginecológica e complementar das pacientes.

\section{Referências}

1. Koike K, Ohno S, Takahashi N, Suzuki N, Nozaki N, Murakami K, et al. Efficacy of the herbal medicine Unkeito as an adjunctive treatment to hormone replacement therapy for postmenopausal women with depressive symptoms. Clin Neuropharmacol. 2004;27(4):157-62.

2. Schechter D. Estrogen, progesterone, and mood. J Gend Specific Med. 1999;2(1):29-36.

3. Schiff R, Bulpitt CJ, Wesnes KA, Rajkumar C. Shortterm transdermal estradiol therapy, cognition and depressive symptoms in healthy older women: A randomized placebo controlled pilot cross-over study. Psychoneuroendocrinology. 2005;30(4):309-15.

4. Haines CJ, Yim SF, Chung TK, Lam CW, Lau EW, $\mathrm{Ng} \mathrm{MH}$, et al. A prospective, randomized, placebocontrolled study of the dose effect of oral oestradiol on menopausal symptoms, psychological well being, and quality of life in postmenopausal Chinese women. Maturitas. 2003;44(3):207-14.

5. Rudolph I, Zimmerman T, Zaminski K, Jandova K, Borovsky B, Ahrendt HJ, et al. Changes in psychic and somatic well-being and cognitive capabilities of peri- and postmenopausal women after use of a hormone replacement therapy containing estradiol valerate and levonorgestrel. Methods Find Exp Clin Pharmacol. 2000;22(1):51-6.

6. Souza RL, Filizola RG, Souza ESS, Moraes JLR. Efeito de hormônios sexuais femininos sobre sintomatologia depressiva no climatério. Reprod Clim. 2001;16(2):118-22.

7. Kaari C, Haidar MA, Soares Júnior JM, Nunes MG, Quadros LG, Kemp C, et al. Randomized clinical trial comparing conjugated equine estrogens and isoflavones in postmenopausal women: a pilot study. Maturitas. 2006;53(1):49-58.

8. Crisafulli A, Marini H, Bitto A, Altavilla D, Squadrito D, Romeo A, et al. Effects of genistein on hot flushes in early postmenopausal women: a randomized, double-blind EPT- and placebo-controlled study. Menopause. 2004;11(4):400-4.

9. Faure ED, Chantre P, Mares P. Effects of a standardized soy extract on hot flushes: a multicenter, double-blind, randomized, placebocontrolled study. Menopause. 2002;9(5):329-34.

10. Hamilton M. A rating scale for depression. J Neurol Neurosurg Psychiatry. 1960;23:56-62.

11. Organização Mundial da Saúde. Classificação de transtornos mentais e de comportamento da CID- 
10: descrições clínicas e diretrizes diagnósticas. Porto Alegre: Artes Médicas; 1993.

12. A decision tree for the use of estrogen replacement therapy on hormone replacement therapy in postmenopausal women: consensus opinion of The North American Menopause Society. Menopause. 2000;7(2):76-86.

13. Moraes JLR. Depressão no Hospital Geral: freqüência de pacientes deprimidos na clientela de internos do Hospital Universitário Lauro Wanderley [dissertação]. Recife: Universidade Federal de Pernambuco; 1996.

14. Associação Brasileira de Empresas de Pesquisa [homepage da Internet]. Critério de classificação econômica Brasil. 2003 [acesso 22 mar 2003]. Disponivel em: http:// www.anep.org.br/codigosguias/ABEP_CCEB.pdf.

15. Liu P, He FF, Bai WP, Yu Q, Shi W, Wu YY, et al. Menopausal depression: comparison of hormone replacement therapy and hormone replacement therapy plus fluoxetine. Chin Med J (Engl). 2004;117(2):189-94.

16. Cohen J. Statistical analysis for the behavioral sciences. Hillsdale: Lawrence Erlbaum; 1988.

17. Federici E, Touche A, Choquart S, Avanti O, Fay L, Offord E, et al. High isoflavone content and estrogenic activity of 25 year-old Glycine max tissue cultures. Phytochemistry. 2003;64(3):717-24.

18. Imwalle DB, Gustafsson JA, Rissman EF. Lack of functional estrogen receptor beta influences anxiety behavior and serotonin content in female mice. Physiol Behav. 2005;84(1):157-63.

19. Rocha BA, Fleisher R, Schaeffer JM, Rohrer SP, Hickey GJ. 17 Beta-estradiol-induced antidepressant-like effect in the forced swim test is absent in estrogen receptorbeta knockout (BERKO) mice. Psychopharmacology. 2005;179(3):637-43.

20. Utian WH. Psychosocial and socioeconomic burden of vasomotor symptoms in menopause: a comprehensive review. Health Qual Life Outcomes. 2005;3(1):47.

21. Gingrich PM, Fogel CI. Herbal therapy use by perimenopausal women. J Obstet Gynecol Neonatal Nurs. 2003;32(2):181-9.

22. Fonseca AM, Bagnoli VR, Cardoso EB, Assis JS. Climatério: abordagem atual do diagnóstico e tratamento. RBM Rev Bras Med. 2004;61(1-2):65-9.

23. Davis SR. Phytoestrogen therapy for menopausal symptoms? BMJ. 2001;323(7309):354-5.

24. Abreu VG, Francischetti EA. Reposição hormonal: controvérsias. RBM Rev Bras Med. 2001;58(4):217-24.

25. Nagata C, Shimizu H, Takami R, Hayashi M, Takeda N, Yasuda K. Serum concentrations of estradiol and dehydroepiandrosterone sulfate and soy product intake in relation to psychologic well-being in periand postmenopausal Japanese women. Metabolism. 2000;49(12):1561-4.

26. Kok L, Kreijkamp-Kaspers S, Grobee DE, Lampe JW, van der Schouw YT. A randomized, placebo-controlled trial on the effects of soy protein containing isoflavones on quality of life in postmenopausal women. Menopause. 2005;12(1):56-62.

27. Morrison MF, Kallan MJ, Tem Have T, Katz I, Tweddy K, Battistini M. Lack of efficacy of estradiol for depression in postmenopausal women: a randomized, controlled trial. Biol Psychiatry. 2004;55(4):406-12.

28. Schmidt PJ, Nieman L, Danaceau MA, Tobin MB, Roca CA, Murphy $\mathrm{JH}$, et al. Estrogen replacement in perimenopause-related depression: a preliminary report. Am J Obstet Gynecol. 2000;183(2):414-20.

29. Secreto G, Chiechi LM, Amadori A, Miceli R, Venturelli E, Valerio T, et al. Soy isoflavones and melatonin for the relief of climacteric symptoms: a multicenter, doubleblind, randomized study. Maturitas. 2004;47(1):11-20.

30. Penotti M, Fabio E, Modena AB, Rinaldi M, Omodei $\mathrm{U}$, Vigano P. Effect of soy-derived isoflavones on hot flushes, endometrial thickness, and the pulsatility index of the uterine and cerebral arteries. Fertil Steril. 2003;79(5):1112-7.

31. Demétrio FN, Reno Júnior J, Gonçalves MA, Gianfaldoni A, Halbe HW, Gorenstein C, et al. Terapia com estrógenos conjugados no humor de mulheres menopausadas: resultados preliminares. Rev Psiquiatr Clin (São Paulo). 2001;28(2):89-93.

32. Rowland IR, Wiseman H, Sanders TA, Adlercreutz $\mathrm{H}$, Bowey EA. Interindividual variation in metabolism of soy isoflavones and lignans: influence of habitual diet on equol production by the gut microflora. Nutr Cancer. 2000;36(1):27-32.

33. Petri-Nahás EA, Nahás-Neto J, De Luca L, Traiman P, Pontes A, Dalben I. Benefits of soy germ isoflavones in postmenopausal women with contraindication for conventional hormone replacement therapy. Maturitas. 2004;48(4):372-80.

34. Han KK, Soares JM Jr, Haidar MA, de Lima GR, Baracat EC. Benefits of soy isoflavone therapeutic regimen on menopausal symptoms. Obstet Gynecol. 2002;99(3):389-94.

35. Huttner RP, Shepherd JE. Gonadal steroids, selective serotonin reuptake inhibitors, and mood disorders in women. Med Clin North Am. 2003;87(5):1065-76.

36. Sousa RL, Filizola RG, Moraes JLR. O efeito dominó dos fogachos: sintomatologia depressiva e insônia no climatério feminino. RBM Rev Bras Med. 2003;60(4):191-4.

37. Baird DD, Umbach DM, Lansdell L, Hughes CL, Setchell $\mathrm{KD}$, Weiberg CR, et al. Dietary intervention study to assess estrogenicity of dietary soy among postmenopausal women. J Clin Endocrinol Metab. 1995;80(5):1685-90.

38. Davis SR, Briganti EM, Chen RQ, Dalais FS, Bailey $\mathrm{M}$, Burger HG. The effects of Chinese medicinal herbs on postmenopausal vasomotor symptoms of Australian women: a randomized controlled trial. Med J Aust. 2001;174(2):68-71.

39. Rajewska J, Rybakowski JK. Depression in premenopausal women: gonadal hormones and serotonergic system assessed by D-fenfluramine challenge test. Prog Neuropsychopharmacol Biol Psychiatry. 2003;27(4):705-9.

40. Albert A, Altabre C, Baro F, Buendia E, Cabero A, Cancelo $\mathrm{MJ}$, et al. Efficacy and safety of a phytoestrogen preparation derived from Glycine max (L.) Merr in climateric symptomatology: a multicentric, open, prospective and non-randomized trial. Phytomedicine. 2002;9(2):85-92. 